\title{
Association of Oxidative Stress and Production of Inflammatory Mediators Matrix Metalloproteinase-9 and Interleukin 6: Systemic Events in Radicular Cysts
}

Arif Malik ${ }^{1}$, Sahar Chawla ${ }^{2}$, Ambreen Tauseef ${ }^{3}$, Hira Sohail ${ }^{2}$, Farhat Ijaz ${ }^{3}$, Aamenah Malik ${ }^{4}$, Farzana Rahman $^{5}$, Ghulam Muhammad ${ }^{6}$, Saad Khakwani ${ }^{7}$

1. Biochemistry, Institute of Molecular Biology and Biotechnology, Lahore University, Lahore, PAK 2. Biochemistry, Combined Military Hospital Lahore Medical College and Institute of Dentistry, Lahore, PAK 3. Physiology, Combined Military Hospital Lahore Medical College and Institute of Dentistry, Lahore, PAK 4. Biochemistry, Combined Military Hospital Lahore Medical College and Institute of Dentistry, Lahore, PSE 5. Orthodontics, Triangle Orthodontics, Chapel Hill, USA 6. Dentistry: Periodontology, Combined Military Hospital Lahore Medical College and Institute of Dentistry, Lahore, PAK 7. Dentistry, Combined Military Hospital Lahore Medical College and Institute of Dentistry, Lahore, PAK

Corresponding author: Ambreen Tauseef, ambertauseef@hotmail.com

\section{Abstract \\ Background}

Matrix metalloproteinase-9 (MMP-9) and antioxidants are associated with the pathogenesis of cysts and may initiate and sustain the formation of new capillaries.

\section{Objective}

The objective of this study was to determine the association of oxidative stress and the production of inflammatory mediators MMP-9 and interleukin 6 (IL-6) in systemic events in radicular cyst growth.

\section{Materials and methods}

Fifty patients (34 men, 16 women) with periapical granulomas and radicular cysts were included in this cross-sectional study. Twenty subjects (12 men, eight women) with no signs of periodontal diseases were recruited as controls. Blood serum levels of MMP-9, IL-6, superoxide dismutase (SOD), malondialdehyde (MDA), and glutathione peroxidase (GPx) were recorded. We also recorded body mass index (BMI) and tumor necrosis factor-alpha (TNF-alpha) levels.

\section{Results}

Received 03/31/2020 Review began 04/09/2020 Review ended 04/16/2020 Published 04/25/2020

\section{() Copyright 2020}

Malik et al. This is an open access article distributed under the terms of the Creative Commons Attribution License CC-BY 4.0., which permits unrestricted use, distribution, and reproduction in any medium, provided the original author and source are credited.
The mean age of the test group patients and control patients was 45.9 and 48.8 years, respectively. The BMI of test group patients $\left(23.77 \pm 3.88 \mathrm{~kg} / \mathrm{m}^{2}\right)$ was higher than that of the controls $\left(27.98 \pm 3.88 \mathrm{~kg} / \mathrm{m}^{2} ; \mathrm{p} \leqslant\right.$ 0.000). Levels of serum MDA ( $p \leqslant 0.033)$, IL-6 $(p \leqslant 0.041)$, TNF-alpha $(p \leqslant 0.004)$, and MMP-9 $(p \leqslant 0.033)$ were significantly increased in patients as compared with control values. SOD $(p \leqslant 0.003)$ and GPx $(p \leqslant 0.033)$ levels were significantly reduced in patients as compared with controls.

\section{Conclusion}

Oxidative imbalance and the increased production of inflammatory mediators may be associated with systemic events in radicular cysts. Bone-resorbing mediators and proinflammatory cytokines that were evaluated in the study (MMP-9, IL-6, C-reactive protein, TNF-alpha) were also elevated in the serum of the ailing group, thus documenting a well-established role for these circulating biochemical variables in the course of the progression and pathogenesis of radicular cyst development.

Categories: Miscellaneous, Infectious Disease, Dentistry

Keywords: antioxidants, inflammatory mediators, radicular cyst

\section{Introduction}

Cyst growth and maturity involves the provision of stimulus by antigens, microbes, fibroblasts, and growth factors that trigger the inflammatory response by releasing various cytokines, ultimately causing explosive cell division. As the epithelial proliferation in a cystic cavity continues, cells in the middle of the mass become deficient of nourishment and begin necrosis. The degenerative tissue and cell debris in the cyst cavity are chemotactic for neutrophils, causing influx into the cyst lumen. The cystic contents have a higher osmotic load as compared to the surrounding tissue serum. 
Moreover, the necrotic and degenerating cells in the interior of the cyst cavity discharge an excessive amount of molecular species, further raising the osmotic pressure of the cyst void, resulting in the flow of liquid from tissues into the cyst lumen. The outcome is an elevated intracystic pressure that can result in osteoclastic bone resorption and cyst enlargement [1-3]. A radicular cyst (RC) is a result of chronic inflammation in response to inflammatory mediators created from the necrosis of dental pulp [4]. According to Khot et al., RCs "derive their epithelial lining from the proliferation of small odontogenic epithelial residues within the periodontal ligament," which contributes to the progression of the lesion [2]. The regulation of extracellular matrix synthesis and degradation is a vital function of metalloproteases (MMPs), which are also involved in embryogenesis, ovulation, development of nerve tissue, vessel formation, apoptosis, and monitoring of various inflammatory mediators, including cytokine cleavage and activation of mediators and defensins. In addition to their involvement in the above-mentioned physiological processes, they are mediators of many infectious and inflammatory diseases, including apical periodontal bone disorders and other periapical lesions [5].

The MMPs predominantly expressed in infectious and necrotic odontogenic lesions are $-1,-2,-3,-8,9$, and 13 [6]. Reactive oxygen species (ROS) signaling may activate MMPs like MMP-2 and MMP-9 and inflammatory mediators, especially in periodontal tissues [7].

Oxidants can cause tissue injury via damage to deoxyribonucleic acid (DNA) and proteins, peroxidative injury to lipid membranes, activation of proinflammatory cytokines, and proteases like MMPs. Oxidative stress has an important role in the pathogenesis of apical periodontitis that can lead to RC formation [8]. Antioxidants present in small concentrations, oppose free radical action, and inhibit or delay substrate oxidation [9].

The mode of action of antioxidants involves both non-enzymatic and enzymatic reactions. Primary antioxidants are superoxide dismutase (SOD), glutathione peroxidase (GPx), and malondialdehyde (MDA) [10-11]. GPx and SOD offset the oxidative effects and prevent damage to cellular DNA [12]. MDA is produced via the peroxidation of polyunsaturated fatty acids, and MDA may reduce the activity of glutathione peroxidase in periapical granulomas [13].

SOD removes superoxide radicals by catalyzing the dismutation of molecular oxygen and hydrogen peroxide. GPx is an oxidant enzyme and requires selenium as a cofactor. In cells, the main role of GPx is to scavenge hydrogen peroxide [14].

Peroxide activation increases the levels of interleukin 6 (IL-6), and MMPs can alter the impact of peroxide [15]. The continuous secretion of proinflammatory cytokines in periodontal tissues (e.g., IL-1, IL-2, IL-6, and tumor necrosis factor-alpha (TNF-alpha)) has been observed. Also, reduced levels of regulatory cytokines (e.g., transforming growth factor-beta-1 and IL-10) have been associated with continued inflammation of the ligaments and supporting tooth structures [16].

This cross-sectional study was designed to determine the association of oxidative stress and the production of inflammatory mediators MMP-9 and IL-6 in systemic events in RC growth.

\section{Materials And Methods}

Fifty patients (34 men, 16 women) aged 20 to 40 years were recruited from the Department of Dentistry, University College of Dentistry, Lahore. Patients with periapical granulomas and RCs were included in the study. These patients had a clinical attachment loss $>3 \mathrm{~mm}$, probing depth $>5 \mathrm{~mm}$, and bleeding on probing. Radiographic evidence of round, well-defined radiolucencies of periapical tissues was required for inclusion. We excluded patients receiving antibiotic therapy (within three months of the start of the study period), those with compromised periodontal status, pregnant and lactating women, and those suffering from any chronic infection or depression. Our control group consisted of 20 subjects (12 men, eight women) with healthy, attached gingiva, no signs of periodontal disease nor bleeding on probing. Our institutional ethical committee approved the study protocol.

We assessed patient body mass index (BMI) and collected blood samples to evaluate MMP-9, IL-6, SOD, MDA, and GPx levels via a chemical assay. Levels of TNF-alpha and IL-6 were measured via enzyme-linked immunosorbent assay.

\section{Statistical analysis}

Study data were analyzed using IBM SPSS Statistics for Windows, Version 21.0 (IBM Corp., Armonk, NY). Variables were shown as mean \pm standard deviation (SD). Patient and control values were compared using the student ' $\mathrm{t}$ ' test. $\mathrm{P} \leqslant 0.05$ was considered significant.

\section{Results}

The mean ages of patients and controls were 45.9 and 48.8 years, respectively. Mean patient BMI (23.77 \pm 
$\left.3.88 \mathrm{~kg} / \mathrm{m}^{2}\right)$ were higher than mean control BMI $\left(27.98 \pm 3.88 \mathrm{~kg} / \mathrm{m}^{2} ; \mathrm{p} \leqslant 0.000\right)$. Levels of serum MDA $(\mathrm{p} \leqslant$ $0.033)$, IL-6 $(p \leqslant 0.041)$, TNF-alpha $(p \leqslant 0.004)$, and MMP-9 $(p \leqslant 0.033)$ were significantly increased in patients as compared to those in the control group (Table 1$)$. However, the levels of SOD $(p \leqslant 0.003)$ and GPx $(p \leqslant 0.033)$ were significantly decreased in patients compared to controls.

\begin{tabular}{|c|c|c|c|}
\hline Variables & Controls ( $(=20)$ & Subjects $(n=50)$ & P-value \\
\hline MDA (nmol/ml) & $1.37 \pm 0.03$ & $4.11 \pm 0.24$ & 0.033 \\
\hline SOD (nmol/ml) & $0.11 \pm 0.03$ & $0.03 \pm 0.01$ & 0.003 \\
\hline GPx (nmol/ml) & $7.87 \pm 1.88$ & $5.88 \pm 1.98$ & 0.033 \\
\hline IL-6 (pg/ml) & $4.98 \pm 0.55$ & $7.78 \pm 1.98$ & 0.041 \\
\hline TNF-a (pg/ml) & $27.98 \pm 4.78$ & $30.87 \pm 5.89$ & 0.004 \\
\hline MMP-9 (pmol) & $44.78 \pm 3.98$ & $166 \pm 6.99$ & 0.033 \\
\hline
\end{tabular}

\section{TABLE 1: Levels of circulating biochemical variables in patients with a periapical cyst}

MDA, malondialdehyde; SOD, superoxide dismutase; IL-6, interleukin-6; TNF-a, tumor necrosis factor-alpha; MMP9, matrix metalloproteinase 9; GPx, glutathione peroxidase

\section{Discussion}

Periodontitis is distinguished by immune-mediated damage of periodontal supporting tissues and loss of teeth. Apical lesions can progress to form an RC, and the RC's level of growth is mainly due to the increased levels of MMP-9 [4].

Our findings of significantly increased levels of serum MDA, IL-6, TNF-alpha, and MMP-9 in patients when compared to controls align with a previous study that suggest RC formation may be due to the degradation of collagen via MMPs (i.e., the main stop in the loss of periodontal supporting tissue) [17]. However, another study suggests the primary factor in RC formation is due to collagenases [18]. The separation of epithelium from the connective tissues results in lesion progression and recurrence [19-20].

Angiogenesis is associated with the degradation of vascular basement membrane and renovation of the extracellular matrix to allow endothelial cells to travel into the surrounding tissues [21]. MMP-9 may have an important role in the angiogenesis of odontogenic cysts. Also, ROS can directly activate MMP-8 and MMP-9 in periodontal tissues via oxidizing enzymes [22]. Oxidative non-proteolytic activation of MMP seems to be important in periodontal inflammation. The exact mechanism of involving MMP-9 in intracellular signaling is unknown, but both MMP-8 and MMP-9 are markers of apical and periodontal disease [23].

An experimental study reported a significant elevation in the levels of MDA, which may be an indicator of oxidative stress [22]. The production of ROS after the activation of polymorphonuclear leukocytes may help in the formation of inflammatory lesions. The imbalance of oxidative species in the periapical part may also help the development of asymptomatic periapical lesions [24].

IL-6 and TNF-alpha mainly modulate the response of the cell during periodontal inflammation, persuade intracellular signaling, and alter the expression of the gene. Signaling molecules like chemokines, cytokines, and growth factors may be handled by the active form of MMPs, regulating their bioavailability and function [25-26]. The imbalance and abundance of cytokines and chemokines play an important role in the tissue changes during the development of periodontitis [27].

According to this study, the levels of SOD and GPx were significantly decreased in patients as compared to controls. The total antioxidant status indicates the ability of antioxidants to scavenge free radicals. The reduced activity of SOD and GPx may further the development of the lesion, resulting in oxidative stress [28]. Non-toxic levels of ROS increase proinflammatory mediators, and enzymes of the extracellular matrix take part in the destruction of apical tissue and the formation of apical lesions [26,29].

Our study was limited by the small sample size. Our findings should be validated in a study with a larger population.

\section{Conclusions}

Increased levels of bone-resorbing mediators and proinflammatory cytokines (e.g., MMP-9, IL-6, C-reactive 
protein, TNF-alpha) have a well-established role in the course of progression of RC development. Moreover, an increase in the lipid peroxidation status (MDA), as well as a sharp rise in the reactive oxygen species (e.g., nitric oxide, advanced oxidation protein products, advanced glycation end-products), plays an important role in the pathogenesis of RC.

\section{Additional Information \\ Disclosures}

Human subjects: Consent was obtained by all participants in this study. Institutional Review Board University of Lahore issued approval IMBB/UOL/15/418. Consent was obtained by all participants in this study. Institutional Review Board University of Lahore issued approval IMBB/UOL/15/418. The Ethical Review Committee considered the ethical aspects of the research proposal entitled " Association of Oxidative stress and production of inflammatory mediators MMP 9 and IL-6: systemic events in radicular cyst.". Animal subjects: All authors have confirmed that this study did not involve animal subjects or tissue. Conflicts of interest: In compliance with the ICMJE uniform disclosure form, all authors declare the following: Payment/services info: All authors have declared that no financial support was received from any organization for the submitted work. Financial relationships: All authors have declared that they have no financial relationships at present or within the previous three years with any organizations that might have an interest in the submitted work. Other relationships: All authors have declared that there are no other relationships or activities that could appear to have influenced the submitted work.

\section{References}

1. Neville BW, Day TA: Oral cancer and precancerous lesions. CA Cancer J Clin. 2002, 52:195-215. 10.3322/canjclin.52.4.195

2. Khot K, Deshmukh SB, Alex S: Comparative analysis of the immunohistochemical expression of vascular endothelial growth factor and matrix metalloproteinase-9 in keratocystic odontogenic tumor, dentigerous cyst and radicular cyst. J Cancer Res Ther. 2015, 11:635-640. 10.4103/0973-1482.144591

3. Pechalova PF, Bakardjiev AG: Cysts of the jaws: a clinical study of 621 cases [Article in Croatian] . Acta Stomatol Croat. 2009, 43:215-224.

4. de Oliveira Rde C, Beghini M, Borges CR, et al.: Higher expression of galectin-3 and galectin-9 in periapical granulomas than in radicular cysts and an increased toll-like receptor- 2 and toll-like receptor- 4 expression are associated with reactivation of periapical inflammation. J Endod. 2014, 40:199-203. 10.1016/j.joen.2013.10.031

5. Stamenkovic I: Extracellular matrix remodelling: the role of matrix metalloproteinases . J Pathol. 2003, 200:448-464. 10.1002/path.1400

6. Belmar MJ, Pabst C, Martínez B, Hernández M: Gelatinolytic activity in gingival crevicular fluid from teeth with periapical lesions. Oral Surg Oral Med Oral Pathol Oral Radiol Endod. 2008, 105:801-806. 10.1016/j.tripleo.2007.12.002

7. Binker MG, Binker-Cosen AA, Gaisano HY, de Cosen RH, Cosen-Binker LI: TGF- $\beta 1$ increases invasiveness of SW1990 cells through Rac1/ROS/NF-KB/IL-6/MMP-2. Biochem Biophys Res Commun. 2011, 405:140-145. 10.1016/j.bbrc.2011.01.023

8. Graves DT, Oates T, Garlet GP: Review of osteoimmunology and the host response in endodontic and periodontal lesions. J Oral Microbiol. 2011, 3:[Epub]. 10.3402/jom.v3i0.5304

9. Halliwell B, Gutteridge JMC: Free Radicals in Biology and Medicine . Oxford University Press, Oxford; 2015. 10.1107/S2059798317004533

10. Carocho M, Ferreira IC: A review on antioxidants, prooxidants and related controversy: natural and synthetic compounds, screening and analysis methodologies and future perspectives. Food Chem Toxicol. 2013, 51:15-25. 10.1016/j.fct.2012.09.021

11. Flohé L: The impact of thiol peroxidases on redox regulation . Free Radic Res. 2016, 50:126-142. 10.3109/10715762.2015.1046858

12. Strycharz-Dudziak M, Kiełczykowska M, Drop B, Swiatek L, Kliszczewska E, Musik I, Polz-Dacewicz M: Total antioxidant status (TAS), superoxide dismutase (SOD), and glutathione peroxidase (GPx) in oropharyngeal cancer associated with EBV infection. Oxid Med Cell Longev. 2019, 2019:5832410. 10.1155/2019/5832410

13. Ayala A, Muñoz MF, Argüelles S: Lipid peroxidation: production, metabolism, and signaling mechanisms of malondialdehyde and 4-hydroxy-2-nonenal. Oxid Med Cell Longev. 2014, 2014:360438. $10.1155 / 2014 / 360438$

14. Molavian H, Madani Tonekaboni A, Kohandel M, Sivaloganathan S: The synergetic coupling among the cellular antioxidants glutathione peroxidase/peroxiredoxin and other antioxidants and its effect on the concentration of H2O2. Sci Rep. 2015, 5:13620. 10.1038/srep13620

15. Cavalla F, Osorio C, Paredes R, et al.: Matrix metalloproteinases regulate extracellular levels of SDF1/CXCL12, IL-6 and VEGF in hydrogen peroxide-stimulated human periodontal ligament fibroblasts. Cytokine. 2015, 73:114-121. 10.1016/j.cyto.2015.02.001

16. Glowacki AJ, Gottardi R, Yoshizawa S, Cavalla F, Garlet GP, Sfeir C, Little SR: Strategies to direct the enrichment, expansion, and recruitment of regulatory cells for the treatment of disease. Ann Biomed Eng. 2015, 43:593-602. 10.1007/s10439-014-1125-2

17. Dahiya P, Kamal R, Gupta R, Bhardwaj R, Chaudhary K, Kaur S: Reactive oxygen species in periodontitis . J Indian Soc Periodontol. 2013, 17:411-416. 10.4103/0972-124X.118306

18. Teronen O, Salo T, Laitinen J, et al.: Characterization of interstitial collagenases in jaw cyst wall . Eur J Oral Sci. 1995, 103:141-147. 10.1111/j.1600-0722.1995.tb00015.x

19. Henriques ÁC, Vasconcelos MG, Galvão HC, de Souza LB, de Almeida Freitas R: Comparative analysis of the immunohistochemical expression of collagen IV, MMP-9, and TIMP-2 in odontogenic cysts and tumors. 
Oral Surg Oral Med Oral Pathol Oral Radiol Endod. 2011, 112:468-475. 10.1016/j.tripleo.2011.05.033

20. Senger DR, Davis GE: Angiogenesis. Cold Spring Harb Perspect Biol. 2011, 3:005090.

10.1101/cshperspect.a005090

21. Davis GE, Stratman AN, Sacharidou A, Koh W: Molecular basis for endothelial lumen formation and tubulogenesis during vasculogenesis and angiogenic sprouting. Int Rev Cell Mol Biol. 2011, 288:101-165. 10.1016/B978-0-12-386041-5.00003-0

22. Franco C, Patricia HR, Timo S, Claudia B, Marcela H: Matrix metalloproteinases as regulators of periodontal inflammation. Int J Mol Sci. 2017, 18:440. 10.3390/ijms18020440

23. Baeza M, Garrido M, Hernández-Ríos P, et al.: Diagnostic accuracy for apical and chronic periodontitis biomarkers in gingival crevicular fluid: an exploratory study. J Clin Periodontol. 2016, 43:34-45. 10.1111/jcpe.12479

24. Dezerega A, Pozo P, Hernández M, et al.: Chemokine monocyte chemoattractant protein-3 in progressive periodontal lesions in patients with chronic periodontitis. J Periodontol. 2010, 81:267-276. 10.1902/jop.2009.090406

25. Korpi JT, Aström P, Lehtonen N, et al.: Healing of extraction sockets in collagenase-2 (matrix metalloproteinase-8)-deficient mice. Eur J Oral Sci. 2009, 117:248-254. 10.1111/j.1600-0722.2009.00620.x

26. Hernández M, Gamonal J, Salo T, Tervahartiala T, Hukkanen M, Tjäderhane L, Sorsa T: Reduced expression of lipopolysaccharide-induced CXC chemokine in Porphyromonas gingivalis-induced experimental periodontitis in matrix metalloproteinase-8 null mice. J Periodontal Res. 2011, 46:58-66. 10.1111/j.16000765.2010.01310.x

27. Stadler AF, Angst PD, Arce RM, Gomes SC, Oppermann RV, Susin C: Gingival crevicular fluid levels of cytokines/chemokines in chronic periodontitis: a meta-analysis. J Clin Periodontol. 2016, 43:727-745. 10.1111/jcpe. 12557

28. Singh AK, Pandey P, Tewari M, Pandey HP, Gambhir IS, Shukla HS: Free radicals hasten head and neck cancer risk: a study of total oxidant, total antioxidant, DNA damage, and histological grade. J Postgrad Med. 2016, 62:96-101. 10.4103/0022-3859.180555

29. Hernández-Ríos P, Pussinen PJ, Vernal R, Hernández M: Oxidative stress in the local and systemic events of apical periodontitis. Front Physiol. 2017, 8:869. 10.3389/fphys.2017.00869 